\title{
Decay Rates of Solutions of an Anisotropic Inhomogeneous $n$-Dimensional Viscoelastic Equation with Polynomially Decaying Kernels
}

\author{
Jaime E. Muñoz Rivera ${ }^{1, \star}$, Eugenio Cabanillas Lapa ${ }^{2, \star \star}$ \\ ${ }^{1}$ National Laboratory for Scientific Computation, Department of Research and Development, Rua \\ Lauro Müller 455, Botafogo Cep. 22290, Rio de Janeiro, RJ, Brasil, and IM, Federal University \\ of Rio de Janeiro \\ ${ }^{2}$ Universidad Nacional Mayor de San Marcos. Av. Venezuela s/n, Lima, Peru
}

Received: 11 August 1994/Accepted: 28 August 1995

Abstract: We consider the anisotropic and inhomogeneous viscoelastic equation and we prove that the first and second order energy decay polynomially as time goes to infinity when the relaxation function also decays polynomially to zero. That is, if the kernel $G_{i j k l}$ satisfies

$$
\dot{G}_{i j k l} \leqq-c_{0} G_{i j k l}^{1+\frac{1}{p}} ; \text { and } G_{i j k l}, G_{i j k l}^{1+\frac{1}{p}} \in L^{1}(\mathbb{R}) \text { for } p>2 \text { such that } 2^{m}-1<p,
$$

then the first and second order energy decay as $\frac{1}{(1+t)^{q}}$ with $q=2^{m}-1$.

\section{Introduction}

Several authors have studied the asymptotic stability of the solutions in viscoelasticity. Thanks to the works $[1-5,8,9,11]$ among others, it is well known that the stability holds for inhomogeneous and anisotropic $n$-dimensional materials and also for one-dimensional nonlinear equations. The question now is about the uniform rate of decay of the solution as time goes to infinity. Somehow, the way that the solution goes to zero depends on the decay of the kernel as time goes to infinity. We may ask, under what conditions on the kernel does the solution decay to zero exponentially or at least polynomially? To fix ideas, let us consider the simplest homogeneous isotropic $n$-dimensional viscoelastic equation with density $\rho=1$,

$$
u_{t t}-\mu \Delta u-(\mu+\lambda) \nabla \operatorname{div} u+\int_{0}^{t} g(t-\tau)[\mu \Delta u-(\mu+\lambda) \nabla \operatorname{div} u] d \tau=0,
$$

where $\lambda$ and $\mu$ stand for Lame's constant and by $g$ we denote the relaxation function. The kernel " $g$ " plays an important role in the study of the asymptotic behaviour of the solutions. To see this, let us cite a few results about the uniform rate of decay. For example, in the work of Hrusa [8] the author showed, among others, properties

$\star$ Supported by a grant of CNPq.

$\star \star$ Supported by a grant of CNPq. 
that the solution of the one dimensional viscoelastic equation goes to zero exponentially when the kernel is given by

$$
g(t)=c_{0} e^{-\gamma t} .
$$

Dassios and Zafiropoulus [6] showed, for the same kernel, that the solution of Eq. (1.1) decays as $t^{-3 / 2}$ for materials which occupy the whole 3-dimensional space, with a rate of decay that can be improved depending on the symmetry of the initial data. The solution of the viscoelastic equation decays uniformly (exponentially in bounded domains and algebraically in $\mathbb{R}^{n}$ ), when the kernel is an exponential function. A serious restriction in this point is that Eq. (1.1) does not behave as a genuine integro differential equation, because in this particular case the integral term can be removed so the resulting equation is a partial differential equation. To see this let us differentiate Eq. (1.1) with respect to time. Using $g^{\prime}(t)=-\gamma g(t)$, we can write, in the resulting equation, the first three terms given in Eq. (1.1) instead of the integral term. So the new equation does not have the integral term; it has turned into a third order (in time) partial differential equation. To know how this transformation is useful let us consider the 3-dimensional Cauchy problem (1.1). Using the Fourier transform we get a third order ordinary differential equation with coefficients depending on the Fourier parameter $\xi$. To know the asymptotic behaviour of the solution we have to study the behaviour of the real part of the roots of the characteristic polynomial associated to the ordinary differential equation. This method works for any ordinary differential equations, with constant coefficients which do not depend on time, but unfortunately does not work for "genuine" integrodifferential equations.

In [10] one of the authors of this paper improved the result due to Dassios and Zafiropoulos. In that paper we proved that, when the kernel decays exponentially to zero, the solution also decays exponentially for bounded domains, while for materials which occupy the whole $\mathbb{R}^{n}$ space, the solution decays as $t^{-n / 2}$, with rates which can be improved depending on the regularity of the initial data. For kernels which decay exponentially, the asymptotic behavior of the solution is known for bounded and unbounded domains. But what about no exponential kernels? More precisely, what can we say when the kernel decays like $(1+t)^{-p}$ ? Does the solution decay exponentially? Does the solution decay algebraically, for example, like $(1+$ $t)^{-q}$ ? If this is the case, then what is the relation between $p$ and $q$ ? It seems to us that there is no result in the literature concerning these questions, so to fill this gap, we will study these points here.

The main result of this paper is to show that a uniform rate of decay also holds for kernels which decay like $(1+t)^{-p}$. We will prove that the solution decay to zero as $(1+t)^{-q}$, where $q=2^{m}-1$ and $p>2^{m}-1$, for some natural number $m$. The rate of decay also depends on the $L^{p}$-regularity of the kernel. This means in our framework that the kernel must satisfy $\int_{0}^{\infty}\left|G_{i j k l}\right| d \tau<\infty$ and $\int_{0}^{\infty}\left|G_{i j k l}\right|^{1-\frac{1}{p}} d \tau<$ $\infty$ and also $\dot{G}_{i j k l} \leqq-C G_{i j k l}^{1+\frac{1}{p}}$ for $2^{m}-1<p$ and $C>0$.

We study in Sect. 2 the asymptotic behaviour of inhomogeneous and anisotropic bounded material, while in Sect. 3 we study the case of materials which occupy the whole $n$-dimensional space. In this later case, to use our approach we consider isotropics and homogeneous materials, because our method uses the Fourier transform of the solution. The hypotheses we consider here are simpler than others use in previous works (see $[2-5,8,9,11]$ ). Our method is different and explores the dissipation given by the memory effect as well as the $L^{p}$-regularity of the kernel, 
to construct a Liapunev functional whose derivative is negative proportional to a power of itself.

We finish this section giving some notations and introducing the hypotheses we need to prove our result. For spatial derivatives we use the following notation:

$$
\mathscr{H}_{i_{1}, \ldots, i_{k}, j_{1}, \ldots, j_{s}}:=\frac{\partial^{s} \mathscr{H}_{i_{1}, \ldots, i_{k}}}{\partial x_{j_{1}} \ldots \partial x_{j_{s}}},
$$

while for time derivatives we use:

$$
\dot{w}:=\frac{\partial w}{\partial t}, \quad \ddot{w}:=\frac{\partial^{2} w}{\partial t^{2}} \quad \text { and } \quad \stackrel{(k)}{w}=\frac{\partial^{k} w}{\partial t^{k}} .
$$

The viscoelastic equation we study in this paper is:

$$
\left.\rho \ddot{u}_{i}=\left\{C_{i j k l} u_{k, l}\right\}_{, j}-\left\{\int_{0}^{t} G_{i j k l}(\cdot, t-\tau) u_{k, l}(\cdot, \tau) d \tau\right\}_{, j} \quad \text { in } \Omega \times\right] 0, T[,
$$

with initial data

$$
u(x, 0)=u_{0}(x), \quad u_{t}(x, 0)=u_{1}(x)
$$

and supporting the Dirichlet boundary condition,

$$
u=0 \quad \text { in } \Gamma \times] 0,+\infty[\text {. }
$$

Our result is also valid for Newman boundary conditions with normalized initial data. The proof is essentially the same. Further, we suppose the following conditions are valid:

$$
C_{i l j k}=C_{j k i l}, \quad G_{i j k l}=G_{k l i j} \quad \text { and } \quad C_{i l j k} \in C^{1}, \quad G_{i j k l} \in C^{3} .
$$

Also there exist positive constants $\alpha, \beta, \kappa, C$ and $v_{0}$ such that

$$
\begin{gathered}
C_{i l j k} w_{i l} w_{j k} \geqq \alpha w_{i l} w_{i l}, \quad G_{i j k l}(\cdot, 0) w_{i l} w_{j k} \geqq \beta w_{i l} w_{i l}, \\
\dot{G}_{l j k l} \leqq-\kappa\left[G_{i j k l}\right]^{1+\frac{1}{p}} ; \quad \ddot{G}_{i j k l} \leqq C\left[G_{i j k l}\right]^{1+\frac{1}{p}}, \quad \text { for } p>1, \\
\int_{\Omega}\left\{C_{i j k l}-\int_{0}^{\infty} G_{i j k l} d \tau\right\} u_{i, j} u_{k, l} d x \geqq v_{0} \int_{\Omega} u_{i, j} u_{i, j} d x .
\end{gathered}
$$

Finally we suppose that the following integrability conditions hold:

$$
\int_{0}^{\infty}\left[G_{i j k l}\right]^{1-\frac{1}{p}}<\infty
$$

for $p>1$.

Remark 1.1. Condition (1.6) says that the kernel is like the function

$$
t \mapsto \frac{1}{(1+t)^{p}}
$$

If we take $p>2$ then hypothesis (1.8) is always true.

In the next section we will prove that the solution of (1.2) decays uniformly to zero as time goes to infinity, for bounded domains. 


\section{Asymptotic Behaviour}

To simplify our analysis we introduce the following notation:

$$
G \square \partial v:=\int_{0}^{t} G_{i j k l}(\cdot, t-\tau)\left\{v_{k, l}(\cdot, t)-v_{k, l}(\cdot, \tau)\right\}\left\{v_{i, j}(\cdot, t)-v_{i, j}(\cdot, \tau)\right\} .
$$

To prove the polynomial rate of decay we will use the following lemma.

Lemma 2.1. Let us suppose that $G$ satisfies hypothesis (1.4) and $G_{i j k l} \in C^{1}$. Then the following identity holds:

$$
\begin{aligned}
& \int_{0}^{t} G_{i j k l}(\cdot, t-\tau) v_{k, l}(\cdot, \tau) d \tau \dot{v}_{i, j} \\
& \quad=-\frac{1}{2} \frac{d}{d t} G \square \partial v+\frac{1}{2} \dot{G} \square \partial v+\frac{1}{2} \frac{d}{d t}\left\{\int_{0}^{t} G_{i j k l} d \tau v_{k, l} v_{i, j}\right\}-G_{i j k l}(\cdot, t) v_{k, l} v_{i, j} .
\end{aligned}
$$

Proof. It is easy to see that

$$
\begin{aligned}
\frac{d}{d t} G \square \partial v & =\frac{d}{d t} \int_{0}^{t} G_{i j k l}(\cdot, t-\tau)\left\{v_{k, l}(\cdot, t)-v_{k, l}(\cdot, \tau)\right\}\left\{v_{i, j}(\cdot, t)-v_{i, j}(\cdot, \tau)\right\} \\
& =\dot{G} \square \partial v+2 \int_{0}^{t} G_{i j k l}(\cdot, t-\tau)\left\{v_{k, l}(\cdot, t)-v_{k, l}(\cdot, \tau)\right\} d \tau v_{i, j} \\
& =\dot{G} \square \partial v+2 \int_{0}^{t} G_{i j k l} d \tau v_{k, l} \dot{v}_{i, j}-2 \int_{0}^{t} G_{i j k l}(\cdot, t-\tau) v_{k, l}(\cdot, \tau) d \tau \dot{v}_{i, j} .
\end{aligned}
$$

From the above it follows that

$$
\begin{aligned}
\frac{d}{d t} G \square \partial v= & \dot{G} \square \partial v+\frac{d}{d t}\left\{\int_{0}^{t} G_{i j k l} d \tau v_{k, l} v_{i, j}\right\}-G_{i j k l} v_{k, l} v_{i, j} \\
& -2 \int_{0}^{t} G_{i j k l}(\cdot, t-\tau) v_{k, l}(\cdot, \tau) d \tau \dot{v}_{i, j},
\end{aligned}
$$

which proves the lemma.

The following lemma plays an important role in the rest of this paper:

Lemma 2.2. Assume that hypotheses of Lemma 2.1 holds. Then for any $w, v \in$ $C\left([0, T] ; L^{2}(\Omega)\right)$, the following inequality is valid:

$$
\begin{gathered}
\int_{\Omega}^{t} \int_{0}^{t} G_{i j k l}(\cdot, t-\tau)\left\{w_{k, l}(\cdot, t)-w_{k, l}(\cdot, \tau)\right\} d \tau v_{l, j} d x \\
\leqq c_{0}\left\{\int_{\Omega} G^{1+\frac{1}{p}} \square \partial w d x\right\}^{\frac{1}{2}}\left\{\int_{\Omega} v_{i, j} v_{i, j} d x\right\}^{\frac{1}{2}} .
\end{gathered}
$$

Proof. Using Holder's inequality we get

$$
\begin{aligned}
& \iint_{\Omega}^{t} G_{i j k l}(\cdot, t-\tau)\left\{w_{k, l}(\cdot, t)-w_{k, l}(\cdot, \tau)\right\} d \tau v_{i, j} d x \\
& \quad=\int_{\Omega} \int_{0}^{t} G_{i j k l}^{\frac{1}{2}-\frac{1}{2 p}} G_{i j k l}^{\frac{1}{2}+\frac{1}{2 p}}(\cdot, t-\tau)\left\{w_{k, l}(\cdot, t)-w_{k, l}(\cdot, \tau)\right\} d \tau v_{i, j} d x
\end{aligned}
$$




$$
\begin{aligned}
& \leqq\left\{\int_{0}^{t} G_{i j k l}^{1-\frac{1}{p}} d \tau\right\}^{\frac{1}{2}} \int_{\Omega}\left\{G^{1+\frac{1}{p}} \square \partial w\right\}^{\frac{1}{2}}\left|v_{i, j}\right| d x \\
& \leqq\left\{\int_{0}^{t} G_{i j k l}^{1-\frac{1}{p}} d \tau\right\}^{\frac{1}{2}}\left\{\int_{\Omega} G^{1+\frac{1}{p}} \square \partial w d x\right\}^{\frac{1}{2}}\left\{\int_{\Omega} v_{i, j} v_{i, j} d x\right\}^{\frac{1}{2}} .
\end{aligned}
$$

From (1.8) we have that the integral:

$$
\int_{0}^{\infty}\left|G_{i j k l}(\cdot, \tau)\right|^{1-\frac{1}{p}} d \tau
$$

is bounded. Taking $c_{0}=\left\{\int_{0}^{t} G_{i j k l}^{1-\frac{1}{p}} d \tau\right\}^{\frac{1}{2}}$ our result follows.

By $E(t ; v)$ we denote

$$
E(t ; v):=\frac{1}{2}\left\{\int_{\Omega} \dot{v}_{i} \dot{v}_{i}+G \square \partial v d x+\int_{\Omega}\left\{C_{i j k l}-\int_{0}^{t} G_{i j k l} d \tau\right\} v_{i, j} v_{k, l} d x\right\} .
$$

Lemma 2.3. Under the above conditions we have

$$
\begin{aligned}
& \frac{d}{d t} E(t ; u)=-\frac{1}{2} \int_{\Omega} G_{i j k l} u_{k, l} u_{i, j} d x+\frac{1}{2} \int_{\Omega} \dot{G} \square \partial u d x, \\
& \frac{d}{d t} E(t ; \dot{u})=-\frac{1}{2} \int_{\Omega} G_{i j k l} \dot{u}_{k, l} \dot{u}_{i, j} d x+\frac{1}{2} \int_{\Omega} \dot{G} \square \partial \dot{u} d x-\int_{\Omega}\left[G_{i j k l}(\cdot, t) u_{k l}(\cdot, 0)\right]_{, j} \ddot{u} d x .
\end{aligned}
$$

Proof. Let us multiply Eq. (1.2) by $\dot{u}_{i}$ to get

$$
\frac{1}{2} \frac{d}{d t}\left\{\int_{\Omega} \dot{u}_{i} \dot{u}_{i}+C_{i j k l} u_{i, j} u_{k, l} d x\right\}=\iint_{\Omega}^{t} G_{i j k l}(\cdot, t-\tau) u_{k, l}(\cdot, \tau) d \tau \dot{u}_{i, j} d x .
$$

Using Lemma 2.1 our first identity holds. To show the second assertion, let us take the time derivative of Eq. (1.2) to get

$$
\stackrel{(3)}{u}_{i}-\left\{C_{i j k l} \dot{u}_{k, l}\right\}_{, j}+\left\{G_{i j k l}(\cdot, 0) u_{k, l}\right\}_{, j}+\left\{\int_{0}^{t} \dot{G}_{i j k l}(\cdot, t-\tau) u_{k, l}(\cdot, \tau) d \tau\right\}_{, j}=0 .
$$

Since $\dot{G}_{i j k l}=-\frac{\partial}{\partial \tau} G_{i j k l}$, we have

$$
\begin{aligned}
\left\{\int_{0}^{t} \dot{G}_{i j k l}(\cdot, t-\tau) u_{k, l}(\cdot, \tau) d \tau\right\}_{, j}= & -\left\{G_{i j k l}(\cdot, 0) u_{k, l}\right\}_{, j}+\left\{G_{i j k l}(\cdot, t) u_{k, l}(\cdot, 0)\right\}_{, j} \\
& +\left\{\int_{0}^{t} G_{i j k l}(\cdot, t-\tau) \dot{u}_{k, l}(\cdot, \tau) d \tau\right\}_{, j} .
\end{aligned}
$$

Substitution of this identity in (2.1) yields:

$$
\stackrel{(3)}{u}_{i}-\left\{C_{i j k l} \dot{u}_{k, l}\right\}_{, j}-\left\{\int_{0}^{t} G_{i j k l}(\cdot, t-\tau) \dot{u}_{k, l}(\cdot, \tau) d \tau\right\}_{, j}=\left\{G_{i j k l}(\cdot, t) u_{k, l}(\cdot, 0)\right\}_{, j} .
$$


Multiplying Eq. (2.2) by $\ddot{u}_{i}$ and using the same reasoning as above, we prove the second part of this theorem.

Remark 2.1. In particular Lemma 2.1 implies that the first and second order energy are bounded, that is:

$$
E(t ; u) \leqq E(0 ; u) ; \quad E\left(t ; u_{t}\right) \leqq c E\left(0 ; u_{t}\right) .
$$

Let us define the functionals $K(t ; v)$ and $I(t ; v)$ as

$$
\begin{aligned}
K(t ; v):= & \frac{1}{2} \int_{\Omega} \ddot{v}_{i} \ddot{v}_{i} d x+\frac{1}{2} \int_{\Omega} C_{i j k l} \dot{v}_{k, l} \dot{v}_{i, j} d x-\int_{\Omega} G_{i j k l}(\cdot, 0) v_{k, l} \dot{v}_{i, j} d x \\
& +\gamma \int_{\Omega} C_{i j k l} v_{k, l} \dot{v}_{i, j} d x-\int_{\Omega} \int_{0}^{t} F_{i j k l}(\cdot, t-\tau) v_{k, l}(\cdot, \tau) d \tau \dot{v}_{i, j} d x, \\
I(t ; v):= & \int_{\Omega} \ddot{v}_{i} \dot{v}_{l} d x-\frac{1}{2} \int_{\Omega} G_{i j k l}(\cdot, 0) v_{k, l} v_{i, j} d x-\int_{\Omega} \int_{0}^{t} \dot{G}_{i j k l} d \tau v_{k, l} v_{i, j} d x+\dot{G} \square \partial v,
\end{aligned}
$$

where

$$
F_{i j k l}:=\gamma G_{i j k l}+\dot{G}_{i j k l} .
$$

with these conditions we get the following lemma

Lemma 2.4. Under the above notations there exist constants $C_{2}, c, \gamma>0$ satisfying

$$
\begin{aligned}
\frac{d}{d t}\{K(t ; u)+(\gamma-c) I(t ; u)\} \leqq & g(t) c E(0)-c \int_{\Omega} \ddot{u}_{i} \ddot{u}_{i} d x-\frac{\beta}{2} \int_{\Omega} \dot{u}_{i, j} \dot{u}_{i, j} d x \\
& -\frac{\gamma}{2} \kappa \int_{\Omega} G_{i j k l}(\cdot, t) u_{k, l} u_{i, j} d x+C_{2} \int_{\Omega} G^{1+\frac{1}{p}} \square \partial u d x
\end{aligned}
$$

Proof. Using the identity

$$
\left\{C_{i j k l} u_{k, l}\right\}_{, j}=\ddot{u}_{i}+\left\{\int_{0}^{t} G_{i j k l}(\cdot, t-\tau) u_{k, l}(\cdot, \tau) d \tau\right\}_{, j},
$$

we get that Eq. (2.1) implies:

$$
\begin{aligned}
\stackrel{(3)}{u}_{i} & -\left\{C_{i j k l} \dot{u}_{k, l}\right\}_{, j}+\gamma \ddot{u}_{i}+\left\{G_{i j k l}(\cdot, 0) u_{k, l}\right\}_{, j}+\gamma\left\{C_{i j k l} u_{k, l}\right\}_{, j} \\
& =-\left\{\int_{0}^{t} F_{i j k l}(\cdot, t-\tau) u_{k, l}(\cdot, \tau) d \tau\right\}_{, j},
\end{aligned}
$$

where $F_{i j k l}$ is given by (2.3). Multiplying (2.4) by $\ddot{u}_{i}$, we have

$$
\begin{gathered}
\frac{1}{2} \frac{d}{d t}\left\{\int_{\Omega} \ddot{u}_{i} \ddot{u}_{i} d x+\int_{\Omega} C_{i j k l} \dot{u}_{k, l} \dot{u}_{i, j} d x\right\}=-\gamma \int_{\Omega} \ddot{u}_{i} \ddot{u}_{i} d x+\int_{\Omega} G_{i j k l}(\cdot, 0) u_{k, l} \ddot{u}_{i, j} d x \\
-\gamma \int_{\Omega} C_{i j k l} u_{k, l} \ddot{u}_{i, j} d x+\int_{\Omega} \int_{0}^{t} F_{i j k l}(\cdot, t-\tau) u_{k, l}(\cdot, \tau) d \tau \ddot{u}_{i, j} d x .
\end{gathered}
$$


Using the identities:

$$
\begin{gathered}
\int_{\Omega} G_{i j k l}(\cdot, 0) u_{k, l} \ddot{u}_{i, j} d x=\frac{d}{d t} \int_{\Omega} G_{i j k l}(\cdot, 0) u_{k, l} \dot{u}_{i, j} d x-\int_{\Omega} G_{i j k l}(\cdot, 0) \dot{u}_{k, l} \dot{u}_{i, j} d x, \\
-\gamma \int_{\Omega} C_{i j k l} u_{k, l} \ddot{u}_{i, j} d x=-\gamma \frac{d}{d t} \int_{\Omega} C_{i j k l} u_{k, l} \dot{u}_{i, j} d x+\gamma \int_{\Omega} C_{i j k l} \dot{u}_{k, l} \dot{u}_{i, j} d x, \\
\int_{\Omega} \int_{0}^{t} F_{i j k l}(\cdot, t-\tau) u_{k, l}(\cdot, \tau) d \tau \ddot{u}_{i, j} d x=\frac{d}{d t} \int_{\Omega} \int_{0}^{t} F_{i j k l}(\cdot, t-\tau) u_{k, l}(\cdot, \tau) d \tau \ddot{u}_{i, j} d x \\
\quad-\int_{\Omega} F_{i j k l}(\cdot, 0) u_{k, l} \ddot{u}_{i, j} d x-\int_{\Omega} \int_{0}^{t} F_{i j k l}(\cdot, t-\tau) u_{k, l}(\cdot, \tau) d \tau \ddot{u}_{i, j} d x,
\end{gathered}
$$

we get

$$
\begin{aligned}
\frac{d}{d t} K(t ; u)= & -\gamma \int_{\Omega} \ddot{u}_{i} \ddot{u}_{i} d x-\int_{\Omega} G_{i j k l}(\cdot, 0) \dot{u}_{k, l} \dot{u}_{i, j} d x+\gamma \int_{\Omega} C_{i j k l} \dot{u}_{k, l} \dot{u}_{i, j} d x \\
& -\int_{0}^{t} \dot{F}_{i j k l}(\cdot, t-\tau)\left\{u_{k, l}(\cdot, t)-u_{k, l}(\cdot, \tau)\right\} d \tau \dot{u}_{i, j} \\
& -\int_{\Omega} F_{i j k l}(\cdot, t) u_{k, l} \dot{u}_{i, j} d x
\end{aligned}
$$

Let us multiply Eq. (2.1) by $\dot{u}$ to get

$$
\begin{aligned}
\frac{d}{d t} \int_{\Omega} \ddot{u}_{i} \dot{u}_{i} d x= & \int_{\Omega} \ddot{u}_{i} \ddot{u}_{i} d x-\int_{\Omega} C_{i j k l} \dot{u}_{k, l} \dot{u}_{i, j} d x+\int_{\Omega} G_{i j k l}(\cdot, 0) u_{k, l} \dot{u}_{i, j} d x \\
& +\int_{\Omega} \int_{0}^{t} \dot{G}_{i j k l}(\cdot, t-\tau) u_{k, l}(\cdot, \tau) d \tau \dot{u}_{i, j} d x .
\end{aligned}
$$

From Lemma 2.1 and recalling the definition of $I$ we obtain:

$$
\frac{d}{d t} I(t ; u)=\int_{\Omega} \ddot{u}_{i} \ddot{u}_{i} d x-\int_{\Omega} C_{i j k l} \dot{u}_{k, l} \dot{u}_{i, j} d x+\int_{\Omega} \dot{G}_{i j k l}(\cdot, t) u_{k, l} u_{i, j} d x+\int_{\Omega} \ddot{G} \square \partial u d x,
$$

which together with relations (2.5) and (2.6) yields:

$$
\begin{aligned}
& \frac{d}{d t}\{K(t ; u)+(\gamma-c) I(t ; u)\}=-c \int_{\Omega} \ddot{u}_{i} \ddot{u}_{i} d x+c \int_{\Omega} C_{i j k l} \dot{u}_{k, l} \dot{u}_{i, j} d x \\
& \quad-\int_{\Omega} G_{i j k l}(\cdot, 0) \dot{u}_{k, l} \dot{u}_{i, j} d x+(\gamma-c) \int_{\Omega} \dot{G}_{i j k l}(\cdot, t) u_{k, l} u_{i, j} d x+(\gamma-c) \int_{\Omega} \ddot{G} \square \partial u d x \\
& \quad-\int_{\Omega} F_{i j k l}(\cdot, t) u_{k, l} \dot{u}_{i, j} d x-\int_{0}^{t} \dot{F}_{i j k l}(\cdot, t-\tau)\left\{u_{k, l}(\cdot, t)-u_{k, l}(\cdot, \tau)\right\} d \tau \dot{u}_{i, j} \cdot
\end{aligned}
$$


Since $C_{i j k l}$ is continuous, there exists a positive constant $c_{0}$ such that

$$
\int_{\Omega} C_{i j k l} \dot{u}_{k, l} \dot{u}_{l, j} d x \leqq c_{0} \dot{u}_{i, j} \dot{u}_{i, j}
$$

From hypothesis (1.5) and taking $c \leqq \frac{\beta}{2 c_{0}}$ we conclude

$$
c \int_{\Omega} C_{i j k l} \dot{u}_{k, l} \dot{u}_{i, j} d x-\int_{\Omega} G_{i j k l}(\cdot, 0) \dot{u}_{k, l} \dot{u}_{i, j} d x \leqq-\frac{\beta}{2} \int_{\Omega} \dot{u}_{i, j} \dot{u}_{k, l} d x .
$$

Using hypothesis (1.6) we get

$$
\begin{gathered}
(\gamma-c) \int_{\Omega} \dot{G}_{i j k l}(\cdot, t) u_{k, l} u_{i, j} d x \leqq-(\gamma-c) \kappa \int_{\Omega} G_{i j k l}^{1+\frac{1}{p}}(\cdot, t) u_{k, l} u_{i, j} d x \\
(\gamma-c) \int_{\Omega} \ddot{G} \square \partial u d x-\int_{\Omega} F_{i j k l}(\cdot, t) u_{k, l} \dot{u}_{i, j} d x \leqq(\gamma-c) C \int_{\Omega} G^{1+\frac{1}{p}} \square \partial u d x \\
+c_{1}\left\{\int_{\Omega} G_{i j k l}(\cdot, t) u_{k, l} u_{i, j} d x\right\}^{\frac{1}{2}}\left\{\int_{\Omega} G_{i j k l}(\cdot, t) \dot{u}_{k, l} \dot{u}_{i, j} d x\right\}^{\frac{1}{2}} ; \\
\int_{0}^{t} \dot{F}_{i j k l}(\cdot, t-\tau)\left\{u_{k, l}(\cdot, t)-u_{k, l}(\cdot, \tau)\right\} d \tau \dot{u}_{i, j} \\
\leqq c_{2}\left\{\int_{\Omega} G^{1+\frac{1}{p}} \square \partial u d x\right\}^{\frac{1}{2}}\left\{\int_{\Omega} \dot{u}_{i} \dot{u}_{i} d x\right\}^{\frac{1}{2}} .
\end{gathered}
$$

From (2.7)-(2.11) and taking $\gamma$ big enough, our conclusion follows. The proof is now complete.

To get our final result we will use the following lemma.

Lemma 2.5. Let us suppose that $f$ is a nonnegative $C^{1}$-function satisfying

$$
f^{\prime}(t) \leqq-k_{0}[f(t)]^{1+\frac{1}{p}}+\frac{k_{1}}{(1+t)^{p}},
$$

then we have that

$$
f(t) \leqq \frac{p^{p} f(0)+2 p^{p-1} c}{\left(p+\sqrt[p]{(g(0)} c_{1} t\right)^{p}}
$$

for $c_{1}:=\kappa_{0} \min \left\{1, \frac{p}{2 \kappa_{0}}\left(\frac{p}{\kappa_{1}}\right)^{1 / p}\right\}$.

Proof. Let us denote by $h(t)$ and $g(t)$ the functions

$$
h(t):=\frac{2 \kappa_{1}}{p(1+t)^{p}}, \quad g(t):=f(t)+h(t) .
$$


We have

$$
\begin{aligned}
g^{\prime}(t) & =f^{\prime}(t)-\frac{2 \kappa_{1}}{(1+t)^{p+1}} \leqq-\kappa_{0}[f(t)]^{1+\frac{1}{p}}-\frac{\kappa_{1}}{(1+t)^{p+1}} \\
& \leqq-\kappa_{0}\left\{[f(t)]^{1+\frac{1}{p}}+\left[\frac{\kappa_{1}}{\kappa_{0}(1+t)^{p}}\right]^{1+\frac{1}{p}}\right\} \\
& =-\kappa_{0}\left\{[f(t)]^{1+\frac{1}{p}}+\frac{p^{1+\frac{1}{p}}}{2 \kappa_{0} \kappa_{1}^{\frac{1}{p}}}[h(t)]^{1+\frac{1}{p}}\right\} .
\end{aligned}
$$

It follows that there exists a positive constant $\kappa_{1}$ for which we have:

$$
g^{\prime}(t) \leqq-\kappa_{1}\left\{[f(t)]^{1+\frac{1}{p}}+[h(t)]^{1+\frac{1}{p}}\right\} \leqq-\kappa_{1}[g(t)]^{1+\frac{1}{p}}
$$

Our result follows.

Lemma 2.6. Let us suppose that the function $G$ satisfies:

$$
\int_{0}^{\infty} G^{1-r} d \tau<\infty
$$

for $r=\frac{2^{m}-1}{p}<1$. Then the following inequality hold:

$$
G \square \partial u \leqq\left\{G^{1-r} \square \partial u\right\}^{\frac{1}{2^{m}}}\left\{G^{1+\frac{1}{p}} \square \partial u\right\}^{1-\frac{1}{2^{m}}}
$$

Proof. Using Holder's inequality we get:

$$
G^{s} \square \partial u \leqq\left\{G^{2 s-1-\frac{1}{p}} \square \partial u\right\}^{\frac{1}{2}}\left\{G^{1+\frac{1}{p}} \square \partial u\right\}^{\frac{1}{2}},
$$

for any $s \geqq 1$, from $(2.13)$ we get:

$$
\begin{aligned}
\underbrace{G}_{s=1} \square \partial u & \leqq \underbrace{\left\{G^{1-\frac{1}{p}} \square \partial u\right\}^{\frac{1}{2}}}_{s=1-\frac{1}{p}}\left\{G^{1+\frac{1}{p}} \square \partial u\right\}^{\frac{1}{2}} \leqq \underbrace{\left\{G^{1-\frac{3}{p}} \square \partial u\right\}^{\frac{1}{4}}}_{s=1-\frac{3}{p}}\left\{G^{1+\frac{1}{p}} \square \partial u\right\}^{\frac{1}{2}+\frac{1}{4}} \\
& \leqq\left\{G^{1-\frac{7}{p}} \square \partial u\right\}^{\frac{1}{8}}\left\{G^{1+\frac{1}{p}} \square \partial u\right\}^{\frac{1}{2}+\frac{1}{4}+\frac{1}{8}},
\end{aligned}
$$

obvious induction shows our result.

Theorem 2.1. Under assumptions of Lemma 2.6 and hypotheses (1.4)-(1.6), for $p>2$ with $2^{m}-1<p$, we have that

$$
E(t ; u)+E(t ; \dot{u}) \leqq c\{E(0 ; u)+E(0 ; \dot{u})\} \frac{1}{(1+t)^{q}},
$$

where $q=2^{m}-1$. 
Proof. Multiplying Eq. (1.2) by $u_{i}$ we get

$$
\begin{aligned}
\frac{d}{d t} \int_{\Omega} \dot{u}_{i} u_{i} d x= & \int_{\Omega} \dot{u}_{i} \dot{u}_{i} d x-\int_{\Omega} C_{i j k l} u_{k, l} u_{i, j} d x \\
& +\int_{\Omega} \int_{0}^{t} G_{i j k l}(\cdot, t-\tau) u_{k, l}(\cdot, \tau) d \tau u_{i, j} d x \\
= & \int_{\Omega} \dot{u}_{i} \dot{u}_{i} d x-\int_{\Omega}\left\{C_{i j k l}-\int_{0}^{t} G_{i j k l} d \tau\right\} u_{i, j} u_{k, l} d x \\
& +\int_{0}^{t} G_{i j k l}(\cdot, t-\tau)\left\{u_{k, l}(\cdot, t)-u_{k, l}(\cdot, \tau)\right\} d \tau u_{i, j}
\end{aligned}
$$

On the other hand, Lemma 2.6 implies

$$
\begin{gathered}
\int_{0}^{t} G_{i j k l}(\cdot, t-\tau)\left\{u_{k, l}(\cdot, t)-u_{k, l}(\cdot, \tau)\right\} d \tau u_{i, j} \\
\leqq \frac{C^{2}}{v_{0}} \int_{\Omega} G^{1+\frac{1}{p}} \square \partial u d x+\frac{v_{0}}{4} \int_{\Omega} u_{i, j} u_{i, j} d x .
\end{gathered}
$$

From (2.14) and (2.15) we get

$$
\frac{d}{d t} \int_{\Omega} \dot{u}_{i} u_{i} d x \leqq \int_{\Omega} \dot{u}_{i} \dot{u}_{i} d x-\frac{3 v_{0}}{4} \int_{\Omega} u_{i, j} u_{i, j} d x+C \int_{\Omega} G^{1+\frac{1}{p}} \square \partial u d x .
$$

From Lemmas 2.1, 2.3, 2.4 and inequality (2.16) positive constants $N_{1}$ and $N_{2}$ exist such that the function

$$
\mathscr{L}(t ; u)=N_{1} E(t ; u)+N_{2} E(t ; \dot{u})+K(t ; u)+(\gamma-c) I(t ; u)+\frac{\beta}{8} c_{p} \int_{\Omega} \dot{u}_{i} u_{i} d x,
$$

satisfies

$$
\begin{aligned}
& \frac{d}{d t} \mathscr{L}(t ; u) \leqq c g(t) \\
& -C_{0}\{\underbrace{\int_{\Omega} u_{i, j} u_{l, j} d x+\int_{\Omega} \dot{u}_{i, j} \dot{u}_{i, j} d x+\int_{\Omega} \ddot{u}_{i} \ddot{u}_{i} d x}_{:=R(t)}+\underbrace{\int_{\Omega}^{\Omega} G^{1+\frac{1}{p}} \square \partial u d x+\int_{\Omega} G^{1+\frac{1}{p}} \square \partial \dot{u} d x}_{:=S(t)}\},
\end{aligned}
$$

where $c_{p}$ denotes Poincaré's constant. Using Lemma 2.6, it is not difficult to see that

$$
S(t) \geqq c\left\{\int_{\Omega} G \square \partial u d x+\int_{\Omega} G \square \dot{u} d x\right\}^{\frac{2^{m}}{2^{m}-1}} ; \quad R(t) \geqq R(t)^{\frac{2^{m}}{2^{m}-1}} .
$$

So we have

$$
\frac{d}{d t} \mathscr{L}(t ; u) \leqq c g(t)+c_{5} \mathscr{L}(t ; u)^{\frac{2^{m}}{2^{m}-1}}
$$

Using Lemma 2.5 our result follows. 


\section{Rates of Decay in $\mathbb{R}^{n}$}

In this section we extend the results due to Dassios and Zafiropoulus [6]. We study the asymptotic behaviour of the energy of the following Cauchy problem:

$$
\begin{gathered}
u_{t t}-\mu \Delta u-(\mu+\gamma) \nabla \operatorname{div} u+\int_{0}^{t} g(t-\tau) \Delta u(\cdot, \tau) d \tau \\
+\int_{0}^{t} h(t-\tau) \nabla \operatorname{div} u(\cdot, \tau) d \tau=0 \quad \text { in } \mathbb{R}^{n}, \\
u(\cdot, 0)=u_{0}(x) ; \quad u_{t}(\cdot, 0)=u_{1}(x) .
\end{gathered}
$$

To do this we prove that the displacement vector field can be decomposed into two parts: solenoidal and irrotational, both having the same regularity as $u$, whose corrsponding energies decay uniformly to zero with the rate depending on the regularity of the initial data and the rate of decay of $g$ and $h$. The regularity of the decompositions is similar to the regularity of the solution for the equation:

$$
\Delta u=f \quad \text { in } \mathbb{R}^{n},
$$

whose existence and regularity results are given in the following lemma

Lemma 3.1. Let $f$ be a $C^{2}$-function satisfying

$$
f \in L^{1}\left(\mathbb{R}^{2}\right) \cap L^{p}\left(\mathbb{R}^{2}\right) \quad \text { and } \quad x \mapsto|x|^{\alpha}|f(x)| \in L^{1}(\mathbb{R}) n=2,
$$

for $p \geqq 2$ and $\alpha>0$. Then there exists a function

$$
u \in C\left(\mathbb{R}^{2}\right) \cap L^{\infty}\left(\mathbb{R}^{2}\right) \text { with } \nabla u \in\left[H^{1}\left(\mathbb{R}^{2}\right)\right]^{2}
$$

satisfying (3.18). While for $n \geqq 3, p>\frac{n}{n-2}$, and $f$ satisfying

$$
f \in L^{1}\left(\mathbb{R}^{n}\right) \cap L^{p}\left(\mathbb{R}^{n}\right),
$$

there exist a solution of (3.18) satisfying:

$$
u \in L^{p}\left(\mathbb{R}^{n}\right) ; \quad \nabla u \in\left[L^{q}\left(\mathbb{R}^{n}\right)\right]^{n} ; \quad \text { for any } q>\frac{n}{n-1} .
$$

In particular we get $\nabla u \in\left[H^{1}\left(\mathbb{R}^{n}\right)\right]^{n}$. Finally if $p>n>2$ we get

$$
u \in L^{p}\left(\mathbb{R}^{n}\right) \cap C\left(\mathbb{R}^{2}\right) \cap L^{\infty}\left(\mathbb{R}^{2}\right) .
$$

Proof. Let us denote by $U$ the function:

$$
U(x)=\left\{\begin{array}{ll}
\frac{1}{2 \pi} \ln (|x|) & \text { if } n=2 \\
\frac{1}{(n-2) \sigma_{n}|x|^{n-2}} & \text { if } n>2
\end{array},\right.
$$

where $\sigma_{n}$ is the area of the unit ball of $\mathbb{R}^{n}$. It is well known that the solution of Eq. (3.18) is given by:

$$
u(x)=\int_{\mathbb{R}^{n}} U(x-\xi) f(\xi) d \xi
$$


Without loss of generality we can suppose that $f$ has bounded support. To prove the regularity result we will use the convolution:

$$
f_{v}=\rho_{v} * f,
$$

where $\rho_{v}$ is the mollifier satisfying

$$
\rho_{v}(-x)=\rho_{v}(x) ; \quad \int_{\mathbb{R}^{n}} \rho_{v}(\xi) d \xi=1 \quad \text { and } \quad \rho_{v}(x)=0, \quad \text { if }|x| \geqq \frac{1}{v} .
$$

It is well known that $f_{v}$ converges to $f$ strong in $L^{r}\left(\mathbb{R}^{n}\right)$ when $f \in L^{r}\left(\mathbb{R}^{n}\right)$. Let us denote by $u_{v}$ the solution of (3.18) for $f=f_{v}$, then it follows that

$$
u_{v}(x)=\int_{\mathbb{R}^{n}} U(x-\xi) f_{v}(\xi) d \xi
$$

From (3.19) it follows that

$$
\begin{aligned}
\int_{\mathbb{R}^{n}}\left|\nabla u_{v}(x)\right|^{2} d x & \leqq \int_{\mathbb{R}^{n}}\left|u_{v} \| f_{v}\right| d x \\
\int_{\mathbb{R}^{n}}\left|\Delta u_{v}(x)-\Delta u_{m}(x)\right|^{2} d x & \leqq \int_{\mathbb{R}^{n}}\left|f_{v}(x)-f_{m}(x)\right|^{2} d x .
\end{aligned}
$$

First, we prove that $u_{v}$ is bounded in $H^{1}\left(\mathbb{R}^{n}\right)$, then that $\left(u_{v}\right)$ is a Cauchy sequence in $C\left(\mathbb{R}^{n}\right) \cap L^{\infty}\left(\mathbb{R}^{n}\right)$. Using (3.19) we have:

$$
\frac{\partial u_{v}}{\partial x_{i}}=\int_{\mathbb{R}^{n}} \frac{\partial U}{\partial x_{i}}(x-\xi) f_{v}(\xi) d \xi
$$

Denoting by $\chi$ and $\chi_{c}$ the characteristic functions over the unit ball and its complementary set respectively, we get

$$
U(\xi)=\chi U(\xi)+\chi_{c} U(\xi) .
$$

It is easy to see that

$$
\frac{\partial U}{\partial x_{i}}(\xi)= \begin{cases}\frac{1}{2 \pi} \frac{\xi_{i}}{|\xi|^{2}} & \text { if } n=2 \\ \frac{-\xi_{i}}{2 \sigma_{n}|\xi|^{n}} & \text { if } n>2\end{cases}
$$

and also

$$
\chi \frac{\partial U}{\partial x_{i}} \in L^{1}\left(\mathbb{R}^{n}\right) \quad \text { and } \quad \chi_{c} \frac{\partial U}{\partial x_{i}} \in L^{r}\left(\mathbb{R}^{n}\right) \text { for any } r>\frac{n}{n-1} .
$$

Using Young's inequality we get:

$$
\chi \frac{\partial U}{\partial x_{i}} * f_{v} \in L^{r}\left(\mathbb{R}^{n}\right) \forall 1 \leqq r \leqq p ; \quad \chi_{c} \frac{\partial U}{\partial x_{i}} * f_{v} \in L^{r}\left(\mathbb{R}^{n}\right) \forall r>\frac{n}{n-1} .
$$

Also,

$$
\begin{gathered}
\left\{\int_{\mathbb{R}^{n}}\left|\chi \frac{\partial U}{\partial x_{i}} * f_{v}\right|^{r} d \xi\right\}^{\frac{1}{r}} \leqq \int_{\mathbb{R}^{n}}\left|\chi \frac{\partial U}{\partial x_{i}}\right| d \xi\left\{\int_{\mathbb{R}^{n}}\left|f_{v}\right|^{r} d \xi\right\}^{\frac{1}{r}} \\
\left\{\int_{\mathbb{R}^{n}}\left|\chi_{c} \frac{\partial U}{\partial x_{l}} * f_{v}\right|^{p} d \xi\right\}^{\frac{1}{p}} \leqq\left\{\int_{\mathbb{R}^{n}}\left|\chi_{c} \frac{\partial U}{\partial x_{i}}\right|^{p} d \xi\right\}^{\frac{1}{p}} \int_{\mathbb{R}^{n}}\left|f_{v}\right| d \xi .
\end{gathered}
$$


Under these conditions, for $p=q=r$, we get that

$$
\int_{\mathbb{R}^{n}}\left|\frac{\partial u_{v}}{\partial x_{i}}\right|^{q} d x \leqq c \int_{\mathbb{R}^{n}}\left|\chi \frac{\partial U}{\partial x_{i}}\right| d \xi \int_{\mathbb{R}^{n}}\left|f_{v}\right|^{q} d \xi+c \int_{\mathbb{R}^{n}}\left|\chi_{c} \frac{\partial U}{\partial x_{i}}\right|^{q} d \xi\left\{\int_{\mathbb{R}^{n}}\left|f_{v}\right| d \xi\right\}^{q},
$$

which implies that

$$
\left\|\frac{\partial u_{v}}{\partial x_{i}}\right\|_{q} \leqq c\left\{\left\|f_{v}\right\|_{q}+\left\|f_{v}\right\|_{1}\right\} \quad \text { for } i=1, \ldots, n,
$$

for $q>n /(n-1)$. To prove that $u_{v}$ is a Cauchy sequence in $C\left(\mathbb{R}^{2}\right) \cap L^{\infty}\left(\mathbb{R}^{2}\right)$, let us consider

$$
\begin{aligned}
\left|u_{v}\right| & =\left|\int_{\mathbb{R}^{n}} U(x-\xi) f_{v}(\xi) d \xi\right|=\left|\frac{1}{\sigma_{2}} \int_{\mathbb{R}^{n}} \ln \right| x-\xi\left|f_{v}(\xi) d \xi\right| \\
& \leqq c_{\alpha} \int_{|\xi| \geqq|x|}|x-\xi|^{\alpha}\left|f_{v}(\xi)\right| d \xi+\left.\frac{1}{\sigma_{2}}\left|\int_{|\xi| \leqq|x|} \frac{\ln |x-\xi|}{|x|^{\alpha}}\right| \xi\right|^{\alpha} f_{v}(\xi) d \xi \mid \\
& \leqq \frac{1}{\sigma_{2}} c_{\alpha}\left|\int_{|\xi| \geqq|x|}\right| x-\left.\left.\xi\right|^{\alpha}\left|f_{v}(\xi)\right| d \xi|+\int_{|\xi| \leqq|x|} \underbrace{\frac{|x-\xi|^{\alpha}}{|x|^{\alpha}}}_{\leqq 2^{\alpha}}| \xi\right|^{\alpha} f_{v}(\xi) d \xi \mid \\
& \leqq C_{\alpha} \int_{\mathbb{R}^{n}}|\xi|^{\alpha}\left|f_{v}(\xi)\right| d \xi .
\end{aligned}
$$

Repeating the above reasoning for $u_{v}-u_{m}$ instead of $u_{v}$, we conclude that $u_{v}$ is a Cauchy sequence in $L^{\infty}\left(\mathbb{R}^{2}\right)$. Since $f$ has bounded support we can apply Lebesgue's Convergence Theorem to show that:

$$
|u| \leqq C_{\alpha} \int_{\mathbb{R}^{n}}|\xi|^{\alpha}|f(\xi)| d \xi .
$$

Using (3.20), (3.21) and the fact that $f \in L^{1}\left(\mathbb{R}^{2}\right) \cap L^{p}\left(\mathbb{R}^{2}\right)$ we conclude that $\nabla u_{v}$ is bounded in $H^{1}\left(\mathbb{R}^{n}\right)$. So, our result follows for case $n=2$. For $n>2$ using (3.22) we get that $\nabla u_{v}$ is bounded in $\left[H^{2}\left(\mathbb{R}^{n}\right)\right]^{n}$. Moreover we have

$$
\chi U \in L^{1}\left(\mathbb{R}^{n}\right), \chi_{c} U \in L^{p}\left(\mathbb{R}^{n}\right) \text { for } p>\frac{n}{n-2} .
$$

From hypotheses on $f$ we get

$$
u_{v} \in L^{q}\left(\mathbb{R}^{n}\right) \cap L^{1}\left(\mathbb{R}^{n}\right)
$$

and also that

$$
\left\{\int_{\mathbb{R}^{n}}\left|u_{v}\right|^{q} d x\right\}^{\frac{1}{q}} \leqq c\left\{\left\|f_{v}\right\|_{q}+\left\|f_{v}\right\|_{1}\right\},
$$

where by $\|\cdot\|_{p}$ we denote the norm of the space $L^{p}$. It remains only to prove the regularity of $u$ when $p>n$. To do this we will use the following lemma:

Lemma 3.2. Let us suppose that $v$ is a continuous function satisfying

$$
u \in L^{q}\left(\mathbb{R}^{n}\right), \quad \nabla u \in\left[L^{p}\left(\mathbb{R}^{n}\right)\right]^{n},
$$


for any $q \geqq 1$ and any $p>n$. Then the following inequality holds:

$$
|v(x)| \leqq \frac{1}{\sqrt[q]{\sigma_{n}}}\|v\|_{q}+\frac{p}{(p-n) \sqrt[p]{\sigma_{n}}}\|\nabla v\|_{p} .
$$

Using Lemma 3.2 we conclude that $u_{v}$ satisfies

$$
\left|u_{v}(x)\right| \leqq c\left\{\left\{\int_{\mathbb{R}^{n}}\left|u_{v}\right|^{q} d x\right\}^{\frac{1}{q}}+\left\{\int_{\mathbb{R}^{n}}\left|\nabla u_{v}\right|^{p} d x\right\}^{\frac{1}{p}}\right\} \leqq C\left\{\left\|f_{v}\right\|_{1}+\left\|f_{v}\right\|_{q}+\left\|f_{v}\right\|_{p}\right\}
$$

and our result follows.

Proof of Lemma 3.2. Let us consider the identity:

$$
v(\xi)-v(x)=\int_{0}^{1} \nabla v(t \xi+(1-t) x) \cdot[\xi-x] d t .
$$

Integration over the ball $B$ of center $x$ and radius $r=1$ yields

$$
\begin{aligned}
\left|\int_{B} v(\xi) d \xi-\sigma_{n} v(x)\right| & \leqq \int_{B} \int_{0}^{1}|\nabla v(t \xi+(1-t) x) \cdot[\xi-x]| d t d \xi \\
& \leqq \int_{B} \int_{0}^{1}|\nabla v(t \xi+(1-t) x)| d t d \xi .
\end{aligned}
$$

Making a change of variable we get

$$
\left|\int_{B} v(\xi) d \xi-\sigma_{n} v(x)\right| \leqq \int_{0}^{1} \frac{1}{t^{n}} \int_{t D}|\nabla v(\xi)| d \xi d t,
$$

where $D$ is the disk of center in the origin of coordinates and the radius equals to $t$. Since $t \leqq 1$ and $t D \subset D$, it follows that

$$
\int_{t D}|\nabla v(\xi)| d \xi d t \leqq \sqrt[p^{\prime}]{\sigma_{n} t^{n}}\left\{\int_{D}\left|\nabla v(\xi)^{p}\right| d \xi\right\}^{\frac{1}{p}}
$$

so we have that:

$$
|v(x)| \leqq \frac{1}{\sigma_{n}}\left|\int_{B} v(\xi) d \xi\right|+\frac{1}{\sqrt[p]{\sigma_{n}}} \frac{p}{p-n}\left\{\int_{D}|\nabla v(\xi)|^{p} d \xi\right\}^{\frac{1}{p}} .
$$

Using the Hölder inequality our conclusion follows.

Now we are in a position to prove the decomposition of the displacement vector field.

Lemma 3.3. Let $f$ be a vector field in $\left[H^{k}\left(\mathbb{R}^{n}\right)\right]^{n}$ such that the divergence of $F$ $(\operatorname{div} F=f)$ satisfies the conditions of Lemma 3.1. Then, we can decompose $F$ in two parts, both in $\left[H^{k}\left(\mathbb{R}^{n}\right)\right]^{n}$, one of them a gradient and the other a solenoidal function (that is with null divergence).

Proof. From Lemma 3.1 there exists a function $p$ such that $\nabla p \in\left[H^{1}\left(\mathbb{R}^{n}\right)\right]^{n}$ satisfies:

$$
\Delta p=\operatorname{div} F, \quad \text { in } \mathbb{R}^{n} .
$$


Since $\Delta p=\operatorname{div} F \in H^{k-1}\left(\mathbb{R}^{n}\right)$, then $\nabla p \in H^{k}\left(\mathbb{R}^{n}\right)$. From the identity:

$$
F=\nabla p+(F-\nabla p)
$$

we obtain the desired decomposition.

Using Lemma 3.3 we can decompose the initial data of Eq. (3.17) in the following way:

$$
u_{0}=u_{0}^{I}+u_{0}^{s} ; \quad u_{1}=u_{1}^{I}+u_{1}^{s},
$$

where $u_{i}^{I}$ and $u_{i}^{s}$ are the irrotational and the solenoidal part of the function $u_{i}$ for $i=1,2$ given in Lemma 3.3. Let us denote by $u^{s}$ by $u^{I}$ the solution of the equations:

$$
\begin{gathered}
u_{t t}^{s}-\mu \Delta u^{s}+\mu \int_{0}^{t} g(t-\tau) \Delta u^{s}(\cdot, \tau) d \tau=0 \quad \text { in } \mathbb{R}^{n}, \\
u(x, 0)=u_{0}^{s}(x), u_{t}(x, 0)=u_{1}^{s}(x), \\
u_{t t}^{I}-(2 \mu+\gamma) \Delta u^{I}+(2 \mu+\gamma) \int_{0}^{t} h(t-\tau) \Delta u^{I}(\cdot, \tau) d \tau=0 \quad \text { in } \mathbb{R}^{n}, \\
u(x, 0)=u_{0}^{I}(x) ; \quad u_{t}(x, 0)=u_{1}^{I}(x),
\end{gathered}
$$

respectively. Since $\operatorname{div} u_{i}^{s}=0$ for $i=1,2$, the solution $u^{s}$ also satisfies: $\operatorname{div} u^{s}=0$. Similarly, since the initial data $u_{i}^{I}$ is such that

$$
\frac{\partial u_{0}^{i, I}}{\partial x_{j}}=\frac{\partial u_{0}^{j, I}}{\partial x_{i}}, \quad \frac{\partial u_{1}^{i, I}}{\partial x_{j}}=\frac{\partial u_{1}^{j, I}}{\partial x_{i}}
$$

then the solution of (3.23) also satisfies:

$$
\frac{\partial u^{i, I}}{\partial x_{j}}=\frac{\partial u^{j, I}}{\partial x_{i}}
$$

Using these properties we conclude that the sum $u^{s}+u^{I}$ is the solution of Eq. (3.17). Our next goal is to prove that the solution of Eq. (3.23) and (3.24) decay algebraically as time goes to infinity. Without loss of generality we consider the equation:

$$
\begin{gathered}
u_{t t}-\mu \Delta u+\mu \int_{0}^{t} g(t-\tau) \Delta u(\cdot, \tau) d \tau=0 \quad \text { in } \mathbb{R}^{n} \\
u(x, 0)=u_{0}(x) ; \quad u_{t}(x, 0)=u_{1}(x)
\end{gathered}
$$

From now on by $g \square v$ we denote:

$$
g \square v=\int_{0}^{t} g(t-\tau)|v(t)-v(\tau)|^{2} d \tau .
$$

Let us define the following functionals:

$$
E(t ; v):=\frac{1}{2} \int_{\mathbb{R}^{n}}\left[|\dot{v}(t)|^{2}+\mu\left(1-\int_{0}^{t} g d \tau\right) \sum_{i=1}^{n}\left|\nabla v_{i}\right|^{2}+\frac{\mu}{2} \sum_{i=1}^{n} g \square \nabla v_{i}\right] d x .
$$


Also we define the density energy function:

$$
\mathscr{E}(\xi, t):=\frac{1}{2}\left|\hat{v}_{t}(t)\right|^{2}+\mu\left(1-\int_{0}^{t} g d \tau\right)|\xi|^{2}\left|\hat{v}_{i}\right|^{2}+\frac{\mu}{2}|\xi|^{2} g \square \hat{v}_{i} .
$$

To get the rate of decay of the energy function we will use the following lemmas:

Lemma 3.4. Let $v$ be a function in $L^{2}\left(\mathbb{R}^{n}\right) \cap L^{1}\left(\mathbb{R}^{n}\right)$ for which there exists $f \in L^{2}\left(\mathbb{R}^{n}\right) \cap L^{1}\left(\mathbb{R}^{n}\right)$ satisfying

Then we have that

$$
v=\partial^{\alpha} f \quad \text { where }|\alpha|=m .
$$

$$
|\hat{v}(\xi)| \leqq[2 \pi]^{\frac{n}{2}}\left|\xi^{\alpha}\right| \int_{\mathbb{R}^{n}}|f(x)| d x \forall \xi \in \mathbb{R}^{n}
$$

Proof. The proof is immediate.

Lemma 3.5. Let $m \geqq 1$ and $s \geqq 0$ be two natural numbers. Then we have that

$$
\int_{0}^{1} \frac{\sigma^{s} d \sigma}{(1+a \sigma)^{m}} \leqq \begin{cases}c(m, s) a^{-m} & \text { if } m \leqq s \\ c(m, s) a^{-(s+1)} & \text { if } m>s+1 \\ c(m, s, \eta) a^{-s+\eta} & \text { if } m=s+1\end{cases}
$$

for $0<\eta<1$, where $c(m, s, \eta) \rightarrow \infty$ as $\eta \rightarrow 0$.

Proof. Let us denote

$$
I(s, m):=\int_{0}^{1} \frac{\sigma^{s} d \sigma}{(1+a \sigma)^{m}}
$$

Integration by parts shows:

$$
\begin{aligned}
\int_{0}^{1} \frac{\sigma^{s} d \sigma}{(1+a \sigma)^{m}} & =\frac{1}{a(-m+1)} \int_{0}^{1} \sigma^{s} d(1+a \sigma)^{-m+1} \\
& =\frac{1}{a(-m+1)}\left[\sigma^{s}(1+a \sigma)^{-m+1}\right]_{\sigma=0}^{\sigma=1}+\frac{s}{a(m-1)} \int_{0}^{1} \frac{\sigma^{s-1} d \sigma}{(1+a \sigma)^{m-1}} \\
& =\frac{1}{a(m-1)} \int_{0}^{1} \frac{\sigma^{s-1} d \sigma}{(1+a \sigma)^{m-1}},
\end{aligned}
$$

so we have:

$$
I(s, m) \leqq \frac{s}{a(m-1)} I(s-1, m-1) .
$$

Note that a simple calculation shows that

$$
\begin{gathered}
I(s, 1) \leqq c(s) a^{-1} \quad \text { for } s \geqq 1, \quad I(0, m) \leqq \frac{1}{m-1} a^{-1} \text { for } m>1, \\
I(0,1)=\frac{\ln (1+a)}{a} .
\end{gathered}
$$

Using the last three inequalities our conclusion follows. 
In this condition we are able to prove the uniform rate of decay of the energy associated to the viscoelastic equation for the whole space $\mathbb{R}^{n}$. Our main result is summarized in the following theorem:

Theorem 3.1. Let us suppose that the functions $g$ and $h$ satisfy the following hypotheses:

$$
\begin{array}{lll}
g(t) \geqq 0 ; & g^{\prime}(t) \leqq-c g^{1+\frac{1}{p}}(t), & g^{\prime \prime}(t) \leqq c g^{1+\frac{1}{p}}(t), \\
h(t) \geqq 0 ; & h^{\prime}(t) \leqq-c h^{1+\frac{1}{p}}(t), & h^{\prime \prime}(t) \leqq c h^{1+\frac{1}{p}}(t),
\end{array}
$$

for $p>2$ such that $2^{m}-1<p$ and the couple $\left(u_{0}, u_{1}\right)$ is a vector field in $\left[H^{2}\left(\mathbb{R}^{n}\right) \cup L^{1}\left(\mathbb{R}^{n}\right)\right]^{n} \times\left[H^{1}\left(\mathbb{R}^{n}\right) \cup L^{1}\left(\mathbb{R}^{n}\right)\right]^{n}$, such that $\operatorname{div} u_{0}$ and $\operatorname{div} u_{1}$ satisfy hypotheses of Lemma 3.3. Then the displacement vector field can be decomposed into two parts, one of them a solenoidal and the other a gradient of a real function whose energy decays as:

where

$$
E(t ; u)+E\left(t ; u_{t}\right) \leqq c\left\{E(0 ; u)+E\left(0 ; u_{t}\right)\right\} \frac{1}{(1+t)^{\gamma}},
$$

$$
\gamma= \begin{cases}2^{m}-1 & \text { if } 2^{m+1} \leqq n-1 \\ \min \left\{2^{m}-1,\left(\frac{1}{2}-\frac{1}{2^{m+1}}\right) n\right\} & \text { if } 2^{m+1}>n-1 \\ \min \left\{2^{m}-1,\left(\frac{1}{2}-\frac{1-\eta}{2^{m+1}}\right)(n-1)\right\} & \text { if } 2^{m+1}=n .\end{cases}
$$

Moreover if there exist functions $f_{0}^{k}, f_{1}^{k} \in L^{2}\left(\mathbb{R}^{n}\right) \cap L^{1}\left(\mathbb{R}^{n}\right)$ such that:

$$
\partial^{\alpha_{k}} f_{0}^{k}=u_{0}^{k}, \quad \text { and } \quad \partial^{\beta_{k}} f_{1}^{k}=u_{1}^{k} .
$$

Then

where

$$
E(t ; u)+E\left(t ; u_{t}\right) \leqq c\left\{E(0 ; u)+E\left(0 ; u_{t}\right)\right\} \frac{1}{(1+t)^{\mu}},
$$

$$
\mu= \begin{cases}2^{m}-1 & \text { if } 2^{m+1} \leqq n-1 \\ \min \left\{2^{m}-1, \frac{2^{m}-1}{2^{m+1}+\beta}(n+\beta)\right\} & \text { if } 2^{m+1}>n-1 \\ \min \left\{2^{m}-1, \frac{2^{m}-1+\beta+\eta}{2^{m+1}+\beta}\left(2^{m}-1\right)\right\} & \text { if } 2^{m+1}=n\end{cases}
$$

with $\beta=\min \left\{\left|\alpha_{k}\right|+1,\left|\beta_{k}\right| ; k=1, \ldots, n\right\}$.

Proof. From Eq. (3.25) we have that the Fourier transform of $u$ satisfies:

$$
\hat{u}_{t t}+|\xi|^{2} \hat{u}-|\xi|^{2} \int_{0}^{t} g(t-\tau) \hat{u}(\cdot, \tau) d \tau=0
$$

Reasoning as in Theorem 2.1 we can prove that:

$$
\frac{d}{d t} \mathscr{E}(\xi, t, \hat{u})=\frac{1}{2}|\xi|^{2} g^{\prime} \square \hat{u}-\frac{1}{2} g(t)|\hat{u}|^{2} .
$$

Differentiating Eq. (3.29) we get

$$
\hat{u}_{t t t}+|\xi|^{2} \hat{u}_{t}-g(0)|\xi|^{2} \hat{u}-|\xi|^{2} \int_{0}^{t} g^{\prime}(t-\tau) \hat{u}(\cdot, \tau) d \tau=0 .
$$


Substituting $\hat{u}$ given by Eq. (3.29) we get:

$$
\hat{u}_{t t t}+|\xi|^{2} \hat{u}_{t}+g(0) \hat{u}_{t t}-|\xi|^{2} \int_{0}^{t} \underbrace{\left[g^{\prime}(t-\tau)+g(0) g(t-\tau)\right]}_{=f(t-\tau)} \hat{u}(\cdot, \tau) d \tau=0 .
$$

Multiplying the above equation by $\hat{u}_{t t}$ we get:

$$
\frac{1}{2} \frac{d}{d t}\left\{\left|\hat{u}_{t t}\right|^{2}+|\xi|^{2}\left|\hat{u}_{t}\right|^{2}\right\}=-g(0)\left|\hat{u}_{t t}\right|^{2}+\underbrace{|\xi|^{2} \int_{0}^{t} f(t-\tau) \hat{u}(\cdot, \tau) d \tau \hat{u}_{t t}}_{=I}
$$

so we have:

$$
\begin{aligned}
I & =|\xi|^{2} \int_{0}^{t} f d \tau \hat{u} \hat{u}_{t t}|\xi|^{2}\left(g(t)-g(0)+g(0) \int_{0}^{t} g d \tau\right) \hat{u} \hat{u}_{t t} \\
& =-|\xi|^{2} g(t) \hat{u} \hat{u}_{t t}+|\xi|^{2} g(0)\left(1-\int_{0}^{t} g d \tau\right) \hat{u} \hat{u}_{t t} .
\end{aligned}
$$

From this identity we get:

$$
\begin{aligned}
I= & -|\xi|^{2} g(t) \hat{u} \hat{u}_{t t}-g(0)|\xi|^{2}\left(1-\int_{0}^{t} g d \tau\right)\left|\hat{u}_{t}\right|^{2}+g(t) g(0)|\xi|^{2} \hat{u} \hat{u}_{t} \\
& +\frac{d}{d t}\left\{|\xi|^{2} g(0)\left(1-\int_{0}^{t} g d \tau\right) \hat{u} \hat{u}_{t}\right\} \\
\leqq & \frac{g(0)}{4}\left|\hat{u}_{t t}\right|^{2}+g(t)|\xi|^{4}|\hat{u}|^{2}+\frac{g(t) g(0)^{2}}{2 \alpha}|\xi|^{2}|\hat{u}|^{2}-\frac{g(0) \alpha}{2}\left|\hat{u}_{t}\right|^{2} \\
& +|\xi|^{2} g(0) \frac{d}{d t}\left\{\left(1-\int_{0}^{t} g d \tau\right) \hat{u} \hat{u}_{t}\right\},
\end{aligned}
$$

where $\alpha=1-\int_{0}^{\infty} g d \tau$. So we conclude that there exist positive constants $N_{1}, N_{2}$ such that the function

$$
\mathscr{L}(\xi, t)=N_{1} \mathscr{E}(\xi, t ; u)+N_{2}|\xi|^{2} \mathscr{E}(\xi, t ; u)\left|\hat{u}_{t t}\right|^{2}+|\xi|^{2}\left|\hat{u}_{t}\right|^{2}-g(0)|\xi|^{2}\left(1-\int_{0}^{t} g d \tau\right) \hat{u} \hat{u}_{t}
$$

satisfies

$$
\frac{d}{d t} \mathscr{L}(\xi, t) \leqq-c_{0}\{\mathscr{R}(\xi, t)+S(\xi, t)\}
$$

where

$$
\mathscr{R}(\xi, t):=\left|\hat{u}_{t t}\right|^{2}+|\xi|^{4}|\hat{u}|^{2}+|\xi|^{2}\left|\hat{u}_{t}\right|^{2}, \quad \mathscr{S}(\xi, t):=|\xi|^{2} g \square \hat{u}+|\xi|^{4} g \square \hat{u},
$$

and

$$
\mathscr{L}(\xi, t) \leqq c_{1} \mathscr{R}(\xi, t)+\mathscr{S}(\xi, t)+c_{2} r(\xi, t)
$$

with

$$
r(\xi, t):=|\xi|^{2}|\hat{u}|^{2}+\left|\hat{u}_{t}\right|^{2}
$$


We will consider two cases. First we prove that the energy density decays algebraically as $\frac{1}{t^{q}}$ for $|\xi| \geqq 1$, then that the density energy function decays as $\frac{1}{t^{\beta}}$ when $|\xi| \geqq 1$. In fact, for $|\xi| \geqq 1$ we get

$$
\mathscr{L}(\xi, t) \leqq c_{3} \mathscr{M}(\xi, t)|\xi|^{2}+|\xi|^{2} g \square \hat{u}+|\xi|^{4} g \square \hat{u} .
$$

Since the energy is bounded, and from Lemma 2.6, a positive constant exists (depending on the initial data) satisfying:

$$
\begin{aligned}
\int_{|\xi| \geqq 1}|\xi|^{2} g \square \hat{u}+|\xi|^{4} g \square \hat{u} d \xi & \leqq C_{1}\left\{\int_{|\xi| \geqq 1}|\xi|^{2} g^{1+\frac{1}{p}} \square \hat{u}+|\xi|^{4} g^{1+\frac{1}{p}} \square \hat{u} d \xi\right\}^{1-\frac{1}{2^{m}}} ; \\
\int_{|\xi| \geqq 1} \mathscr{L}(\xi, t) d \xi & \leqq c_{1}\left\{\int_{|\xi| \geqq 1} \mathscr{R}(\xi, t)+\mathscr{S}(\xi, t) d \xi\right\}^{1-\frac{1}{2^{m}}} .
\end{aligned}
$$

We get:

$$
\frac{d}{d t} \int_{|\xi| \geqq 1} \mathscr{L}(\xi, t) d \xi \leqq-c_{0}\left\{\int_{|\xi| \geqq 1} \mathscr{L}(\xi, t) d \xi\right\}^{\frac{2^{m}}{2^{m}-1}},
$$

which implies that

$$
\int_{|\xi| \geqq 1} \mathscr{L}(\xi, t) d \xi \leqq \frac{\int_{|\xi| \geqq 1} \mathscr{L}(\xi, 0) d \xi}{\left\{q+c_{0}\left(\int_{|\xi| \geqq 1} \mathscr{L}(\xi, 0) d \xi\right)^{\frac{1}{p}} t\right\}^{q}},
$$

so, we have

$$
\int_{|\xi| \geqq 1} \mathscr{L}(\xi, t) d \xi \leqq c \int_{|\xi| \geqq 1} \mathscr{L}(\xi, 0 ; \hat{u}) d \xi \frac{1}{(1+t)^{q}} .
$$

Now we consider the case $|\xi| \leqq 1$. Since

$$
|\xi|^{2} \mathscr{L}(\xi, t ; \hat{u}) \leqq c_{3}\{\mathscr{R}(\xi, t ; \hat{u})+\mathscr{S}(\xi, t)\}^{1-\frac{1}{2^{m}}} \quad \forall|\xi| \leqq 1,
$$

it follows that

$$
\frac{d}{d t} \mathscr{L}(\xi, t) \leqq-c_{0}\left\{|\xi|^{2} \mathscr{L}(\xi, t)\right\}^{\frac{2^{m}}{2^{m-1}}}
$$

which implies

$$
\mathscr{L}(\xi, t ; \hat{u}) \leqq c \frac{1}{\left(\mathscr{L}(\xi, 0)^{-\frac{1}{q}}+|\xi|^{r} t\right)^{q}} .
$$

Since the initial data belongs to $L^{1}\left(\mathbb{R}^{n}\right)$, and $\hat{u}$ is a continuous function, then we get

$$
\mathscr{L}(0, \xi) \leqq c \quad \forall|\xi| \leqq 1 .
$$

From (3.32) a positive constant $c$ exists satisfying:

$$
\mathscr{L}(\xi, t ; \hat{u}) \leqq \frac{c}{\left(1+|\xi|^{r} t\right)^{q}},
$$


where $r:=2+\frac{2}{q}=\frac{2^{m+1}}{2^{m}-1}$. It is easy to see that:

$$
\mathscr{L}(\xi, t ; \hat{u}) \leqq \frac{c_{0}}{\left(1+|\xi| t^{\frac{1}{r}}\right)^{2^{m+1}}} .
$$

Using spherical coordinates we conclude that

$$
\int_{|\xi| \leqq 1} \mathscr{L}(\xi, t ; \hat{u}) d \xi \leqq c \int_{0}^{1} \frac{\sigma^{n-1} d \sigma}{\left(1+t^{\frac{1}{r}} \sigma\right)^{2^{m+1}}},
$$

where $n$ is the space dimension. Using Lemma 3.5 our conclusion follows. Finally if hypothesis (3.28) holds, then a positive number $\beta$ exists such that $\mathscr{L}(0, \xi) \leqq c|\xi|^{\beta}$, so we get,

$$
\mathscr{L}(\xi, t ; \hat{u}) \leqq c \frac{|\xi|^{\beta}}{\left(1+|\xi|^{r+\frac{\beta}{q}} t\right)^{q}} \leqq \frac{|\xi|^{\beta}}{\left(1+|\xi| t^{\gamma}\right)^{2^{m+1}+\beta}}
$$

wherre $\gamma:=\frac{q}{q^{r}+\beta}=\frac{2^{m}-1}{2^{m+1}+\beta}$. Again using Lemma 3.5 our conclusion follows. The proof is now complete.

Remark 3.1. Theorems 2.1 and 3.1 are optimal in the sense that when $m$ increases, the rate of decay of the energy also increases. In the limit case when $m=\infty$, $(p=\infty)$ from the hypotheses we get that the kernel of convolution is exponential. In this case our result is the same as in [10] or in [6].

\section{References}

1. Mac Camy, R.C.: A model for one dimensional non linear Viscoelasticity. Quart. Appl. Math. 35, 21-33 (1977)

2. Dafermos, C.M.: An abstract Volterra Equation with application to linear Viscoelasticity. J. Differ. Eq. 7, 554-589 (1970)

3. Dafermos, C.M.: Asymptotic Stability in Viscoelasticity. Arch. Rat. Mech. Anal. 37, 297-308 (1970)

4. Dafermos, C.M., Nohel, J.A.: Energy method for nonlinear hyperbolic Volterra integrodifferential equation. Comm. PDE 4, 219-278 (1979)

5. Dafermos, C.M., Nohel, J.A.: A nonlinear hyperbolic Volterra equation in Viscoelasticity. Amer. J. Math Supplement 87-116 (1981)

6. Dassios, G., Zafiropoulos, F.: Equipartition of energy in Linearized 3-D Viscoelasticity. Quart. Appl. Math 48, No. 4, 715-730 Dez 1990

7. Greenberg, J.M.: A priori estimates for flows in dissipative materials. J. Math. Anal. Appl. 60, 617-630 (1977)

8. Hrusa, W.J.: Global existence and asymptotic stability for a semilinear Volterra equation with large initial data. SIAM J. Math Anal. 16, No. 1, January 1985

9. Hrusa, W.J., Nohel, J.A.: The Cauchy problem in one dimensional nonlinear Viscoelasticity. J. Diff. Eq. 58, 388-412 (1985)

10. Muñoz Rivera, J.E.: Asymptotic behaviour in Linear Viscoelasticity. Quarterly of Applied Mathematics 3, No. 4, 629-648 (1994)

11. Renardy, M., Hrusa, W.J., Nohel, J.A.: Mathematical problems in Viscoelasticity. Pitman monograph in Pure and Applied Mathematics 35, 1987 